\title{
Biopolymer-Surfactant Complexes as Flow Enhancers: Characterization and Performance Evaluation
}

https://doi.org/10.1515/arh-2019-0002

Received Sep 24, 2018; accepted Feb 07, 2019

\begin{abstract}
Artificial polymeric additives are known, and experimentally proven, to be effective drag reducing agents in pipelines with turbulent flow medium. The artificial nature of these additives and their low resistance to high shear forces, exerted by the pipeline geometries and equipment, are considered as major problems against a wider implementation in other industrial applications. The present work introduces a new polymer-surfactant complex of two organic additives (chitosan and sodium laurel ether sulfate, SLES) as a drag reducing agent. The rheological and morphological properties of the new complexes were experimentally tested. The new complex's drag reduction performance and stability against high shear forces were analyzed using rotating disk apparatus. All the investigated solutions and complexes showed a non-Newtonian behavior. The cryo-TEM images showed a unique polymer-surfactant macrocomplex structure with a nonlinear relationship between its rheological properties and surfactant concentration. A maximum flow enhancement of $47.75 \%$ was obtained by the complex (chitosan 300 and 400 ppm of chitosan and SLES, respectively) at the rotation speed of $3000 \mathrm{rpm}$. Finally, the stability of the proposed additives was highly modified when the additive complexes were formed.
\end{abstract}

Keywords: Drag reduction; Viscoelasticity; Organic polymers; Turbulence; Polymer-surfactant complexes

PACS: 83.60.Yz, 83.60.Bc, 47.50.-d

\footnotetext{
`Corresponding Author: Hayder A. Abdulbari: Faculty of Chemical and Natural Resources Engineering, University of Malaysia Pahang, Lebuhraya Tun Razak, 26300 Gambang, Kuantan, Pahang, Malaysia; Center of Excellence for Advanced Research in Fluid Flow, University of Malaysia Pahang, Lebuhraya Tun Razak, 26300 Gambang, Kuantan, Pahang, Malaysia; Email: abhayder@ump.edu.my Wafaa K. Mahmood, Eman E.: Department of Production Engineering and Metallurgy, University of Technology-IRAQ, Baghdad, Iraq
}

\section{Introduction}

Flow enhancement (drag reduction) in pipelines attracts the attention of many researchers around the globe due to its high academic and industrial impact [1-6]. Over the past few decades, several drag reduction techniques were introduced and experimentally investigated in different applications such as in the Alaskan pipeline [7-9], the transportation of suspensions and slurries, in marine systems and medical fields $[10,11]$. In many cases, the addition of viscoelastic polymeric additives to the core turbulent flow in pipelines resulted in massive reduction of drag. The effectiveness of the additive was controlled by many important parameters such as the liquid's physical properties, pipe dimensions, and the additive's molecular weight [12-15]. Most of the experimentally applied drag reducing agents (DRA) consist of artificial polymeric additives base with high molecular weights (more than 1 million). The use of these polymers has several advantages and disadvantages. It is believed that the high molecular weight polymeric DRA can reduce the drag massively at a very low concentration (in ppm) which is a financially feasible [16-18]. However, most of the known polymeric DRAs are artificial, non-biodegradable, not environmentally friendly and often toxic ,which limits their applications to the crude oil transportation industry only. In addition, the resistance of these long-chained high molecular weight polymers toward mechanical shear forces exerted by the pipe's joints and pumping stations is very low and irreversible [19]. In most cases, when the polymeric solutions are exposed to high shear forces, the polymeric molecules tend to break to smaller molecules or even monomers, which reduces or eliminates their drag reduction effectiveness [20]. Organic polymers are successfully implemented in the fields of food industry and cosmetics $[25,26]$. A few attempts were made by Abdulbari et al. $[27,28]$ to utilize these additives as effective drag reducing

Wafaa A. Khadum: Petroleum Technology Department, University of Technology, Baghdad, Iraq

つopen Access. @ 2019 W. K. Mahmood et al., published by De Gruyter. (cc) BY 4.0 License 
agents in pipelines. Several types of additives from different plants were successfully investigated as DRA such as aloe vera [29], hibiscus leaves [30, 31], and okra [27, 28]. Despite the excellent drag reduction performance of the proposed organic polymers, all these additives showed low stability against shear forces exerted by pumps, valves and joints.

Polymer-surfactant systems have been widely investigated and applied in different fields such as medical, cosmetics and food industries [32]. The stabilization of these complexes usually occurs by the electrostatic interactions between the surfactant charged head and the polymer charged chain and also between the polymer backbone and the surfactant alkyl tail. These complexes (aggregates) usually appear in different formations depending on the nature of the surfactants and polymers themselves.Some of these formations look cubical [33], lamellar [34] or even hexagonal [35]. The creation of polymer-surfactant complexes is a very promising approach towards enhancing the drag reduction performance of the additives and enhancing their resistance to high shear forces. Although the method is purely physical and less complex than the chemical modification procedure, it is still not well explored and identified. The aim of the present work is to introduce a new organic polymer-surfactant complex as drag reducing agent. Cationic chitosan solution and its complexes with an anionic sodium laurel ether sulfate (SLES) surfactant were synthesized and characterized rheologically and morphologically as the main DRA. The solution's stability against high shear forces and its drag reduction performance were tested using rotating disk apparatus (RDA).

\section{Methodology}

\subsection{Preparation of solutions}

Commercial chitosan with the molecular structure $\mathrm{C}_{56} \mathrm{H}_{103} \mathrm{~N}_{9} \mathrm{O}_{39}$ and molecular weight of $1562 \mathrm{~g} / \mathrm{mole}$ was used without any further purification as the main polymeric drag reducing agent. The chitosan-pure water solution was prepared by dissolving the chitosan in $1 \%$ acetic acid-water solution $(\mathrm{pH}=6.3)$ with continuous stirring using magnetic stirrer for 3 hours until complete dissolution was achieved [33]. The SLES surfactant (purchased from Sigma Aldrich) was used without any further modification. The concentrations of chitosan solutions investigated were $50,150,300,600$, and $800 \mathrm{ppm}$ while the SLES surfactant concentration was fixed at $400 \mathrm{ppm}$. The mixture of chitosan and SLES was stirred for 1 hour to ensure complete mixing. Then the mixture was left for $12 \mathrm{~h}$ at $24-25^{\circ} \mathrm{C}$ before testing.

\subsection{Rheology of the solutions}

The rheological properties of all the solutions were measured using a rheometer (Malvern Kinexus Lab+). The viscosity of the DRA solution was measured with a shear rate ranging from 10 to $800 \mathrm{~s}^{-1}$ using the cone-plate geometry (CP2/60 SR22750SS). The analysis to determine viscoelasticity properties of the additives solutions was conducted at frequencies ranging from 0.1 to $100 \mathrm{~Hz}$ using the parallel-plate geometry (PU60 SR3192 SS).

\subsection{Morphology of the solutions}

The morphology of pure chitosan, pure SLES, and their aggregates solutions were investigated using the cryogenic transmission electron microscopy (cryo-TEM) technique atthe Center of Excellence for Advanced Research in Fluid Flow (CARIFF), Universiti Malaysia Pahang.

\subsection{Drag reduction and stability of the solutions}

The drag reduction and stability tests were conducted using rotating disk apparatus (RDA) [37, 38]. A full description of the RDA apparatus is in the supplementary file. The drag reduction performance was determined by comparing the torque readings before and after the introduction of additives. The drag reduction (\%DR) was calculated as follows:

$$
\% D R=\frac{T_{b}-T_{a}}{T_{b}} \times 100
$$

where $\mathrm{T}_{a}$ is the torque reading for additive solutions and $\mathrm{T}_{b}$ is the torque reading for additive-free liquid.

The solutions stability against shear forces was determined for selected samples (pure water, 300 ppm of chitosan, $400 \mathrm{ppm}$ of SLES, and their complexes) by applying $3000 \mathrm{rpm}$ of shear force for 800 seconds. The torque readings were monitored throughout the process. The exposure time was minimized to avoid heating due to the high shear force applied and the temperature was kept at $22-22.3^{\circ} \mathrm{C}$. 


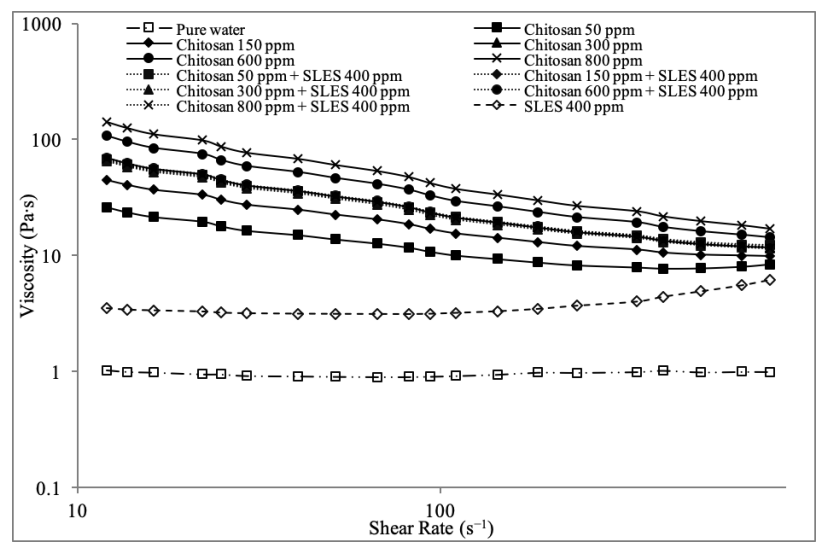

Figure 1: Viscosity of chitosan, SLES, and chitosan-SLES complexes compared to pure water as a function of shear rate

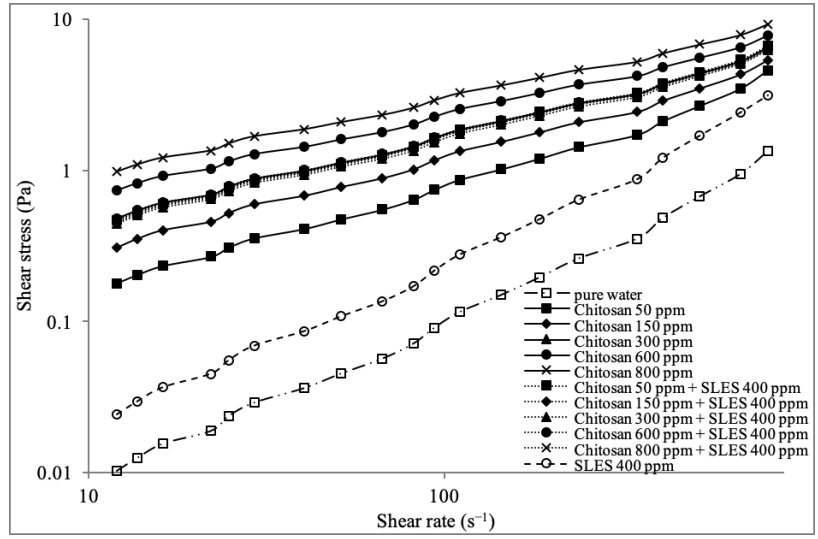

Figure 2: Shear stress of chitosan, SLES, and chitosan-SLES complex solutions compared to pure water as a function of shear rate

\section{Results and Discussions}

Figures 1 and 2 show the rheological properties of the investigated individual solutions, clearly indicating that all the investigated solutions showed a non-Newtonian behavior. In Figure 1, the chitosan solutions exhibited a shear thinning behavior while the SLES solution showed a shear thickening property. Generally, polymers exhibit shear thinning properties because the viscosity of the fluids decreases with increasing shear rate due to the disentanglement and alignment of the polymer chains during high shear rate. In this case, the fluid is also known as pseudoplastic [39-41].

The viscosity of the complex solutions was higher than that of the pure SLES solutions but lower than the viscosity of the high chitosan concentration solutions (more than $300 \mathrm{ppm}$ ). The pure chitosan solution showed a pseudoplastic non-Newtonian behavior and the values of viscosity and shear stress increased linearly with the addi- tive concentration. This relationship was not linear for the chitosan-SLES complexes. The experimental data clearly shows that the viscosity of all the complex solutions was close to the $300 \mathrm{ppm}$ chitosan zone. The addition of SLES did not enhance the viscosity of the chitosan solution, but it remained higher than the viscosity of SLES itself and the low chitosan concentrations (50 and $150 \mathrm{ppm}$ ). The same behavior was observed in the shear stress parameter, which indicates that the effect of combining two different additives (polymer and surfactant) was not rheologically cumulative and the new aggregates had completely different properties given that the effect of the SLES addition was also not linear for the rheological properties of the complex. The complex of $300 \mathrm{ppm}$ of chitosan and 400 ppm of SLES had the highest viscosity and shear stress, while increasing the chitosan concentration (up to 800 $\mathrm{ppm}$ ) did not affect the rheological properties of the resulting complexes solutions.

The viscoelastic properties of the chitosan-SLES complexes are presented in Figure 3. Viscoelasticity measurements demonstrate the properties of a material with both viscous and elastic behavior when stress is applied. The measurements are explained in terms of storage modulus $\left(\mathrm{G}^{\prime}\right)$ and loss modulus $\left(\mathrm{G}^{\prime \prime}\right)$. The storage modulus describes the elastic properties of a fluid in which energy can be stored in the elastic structure under deformation. Meanwhile, the loss modulus shows the viscous behavior of a sample and explains the energy that dissipates as heat. When $\mathrm{G}^{\prime}$ dominates, the fluid behaves as liquid-like while higher $\mathrm{G}^{\prime \prime}$ indicates a solid-like property.

From the results, $G^{\prime \prime}$ dominated at lower frequency proving that the complex solution had viscous behavior. Increasing the frequency resulted in the crossover between $G^{\prime}$ and $G^{\prime \prime}$ confirming the viscoelastic properties of the DRA. As shown in Figure 3a, the crossover point occurred at around $25 \mathrm{~Hz}$. Beyond this point, $\mathrm{G}^{\prime}$ dominated showing an elastic behavior and perhaps a weak gel structures [42]. Also, when the concentration of the chitosan was increased to $300 \mathrm{ppm}$ (Figures $3 \mathrm{~b}$ and 3c), the crossover point occurred at around $20 \mathrm{~Hz}$. However, the crossover started at a lower frequency of $15 \mathrm{~Hz}$ for complexes with higher concentration of chitosan (600 and 800 ppm). All the solutions showed a distinct viscoelastic behavior as expected given that this property is responsible for the flow enhancement process.

The cryo-TEM images of the 300 ppm chitosan, 400 ppm SLES, and their complexes are presented in Figure 4. Distinct dense semi-cubic shapes were observed in the chitosan aggregates (Figure 4a) while the SLES aggregates shapes were completely spherical (Figure 4b). Figure 4c shows the cryo-TEM image of the chitosan-SLES complex 


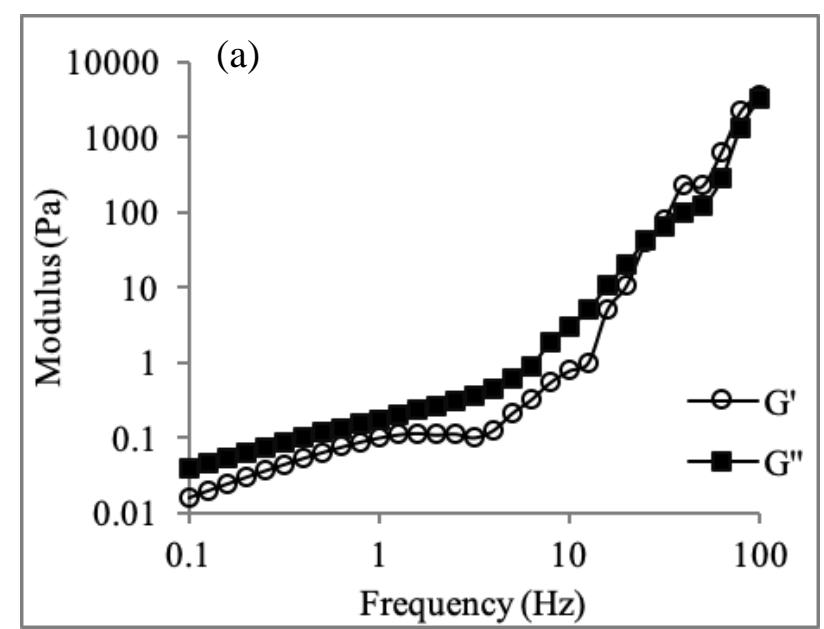

(a)

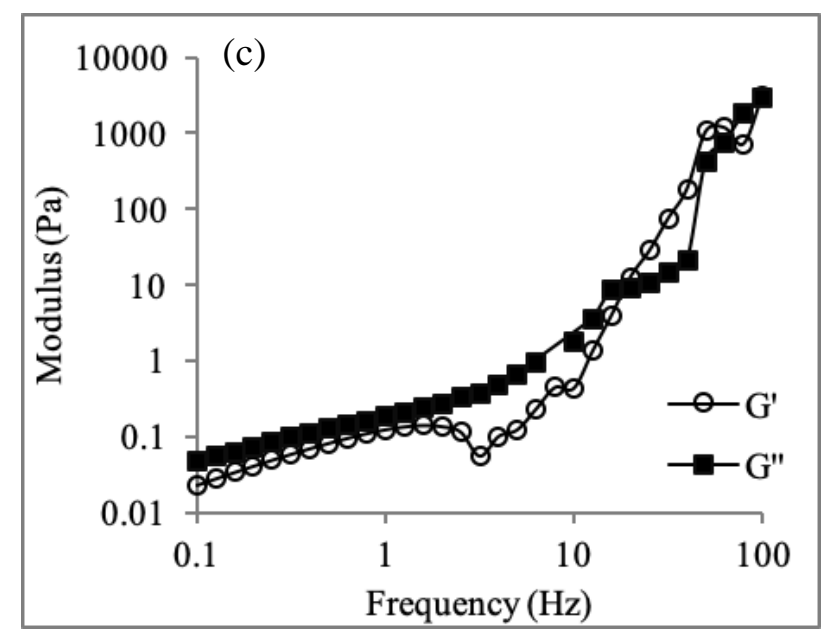

(c)

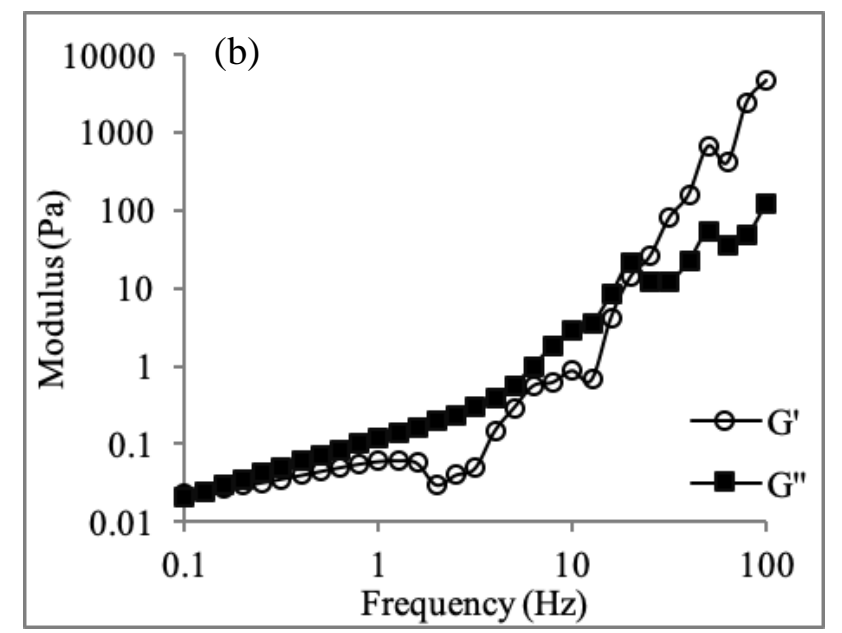

(b)

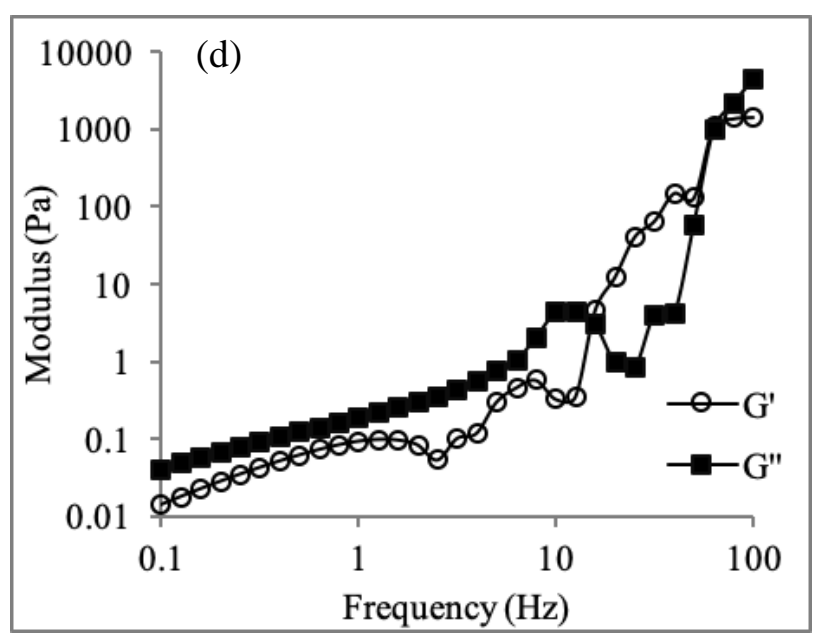

(d)

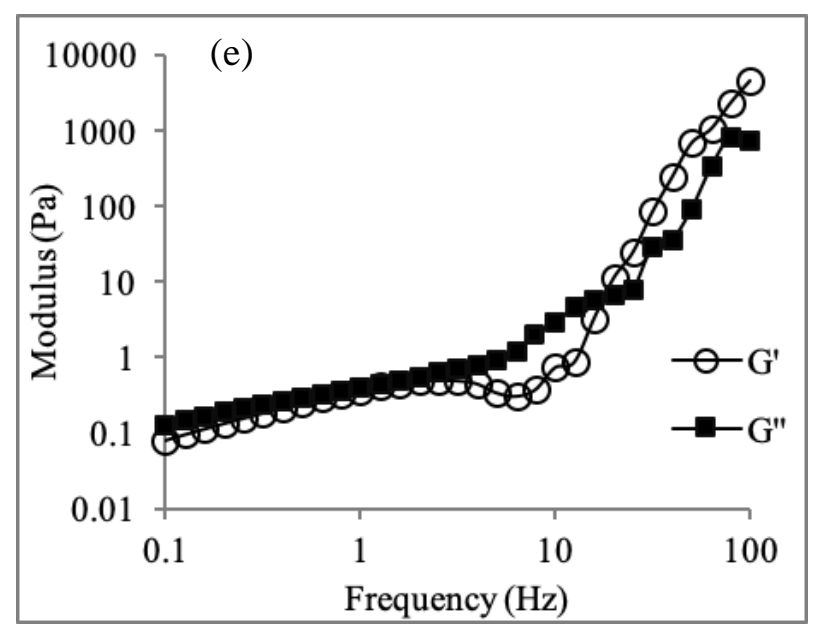

(e)

Figure 3: Viscoelasticity curves of the chitosan-SLES complexes at the concentrations of (a) 50-400 ppm, (b) 150-400 ppm, (c) 300-400 ppm, (d) 600-400 ppm, and (e) 800-400 ppm as a function of sweep frequency 


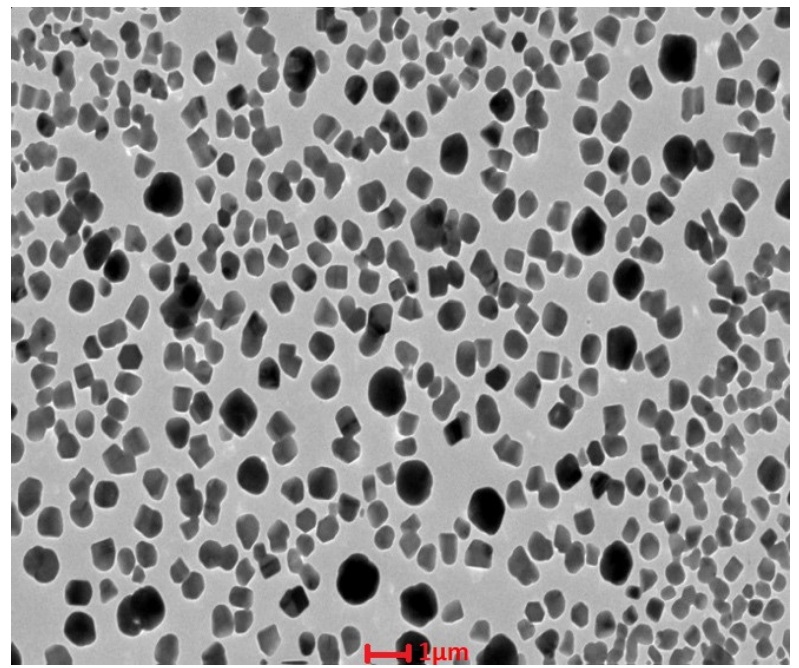

(a)

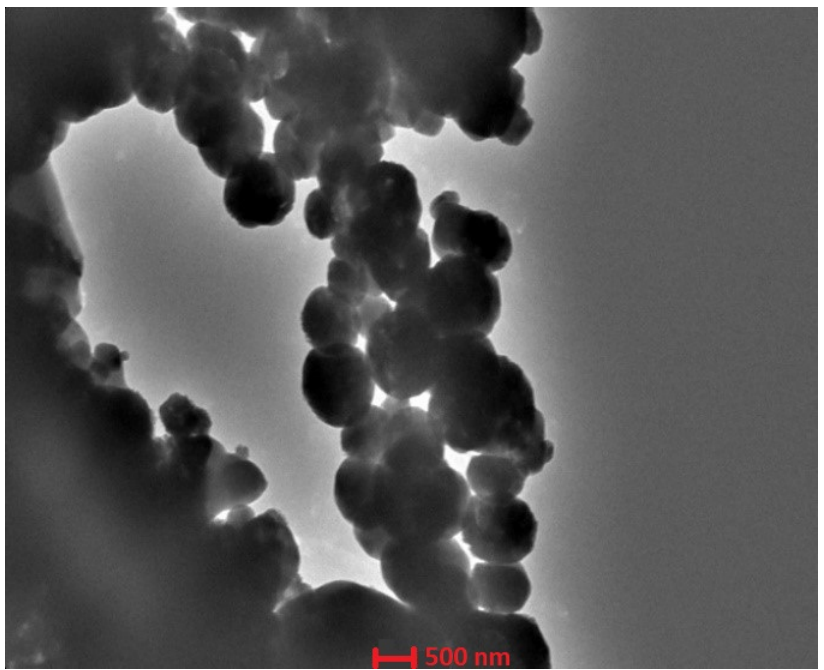

(b)

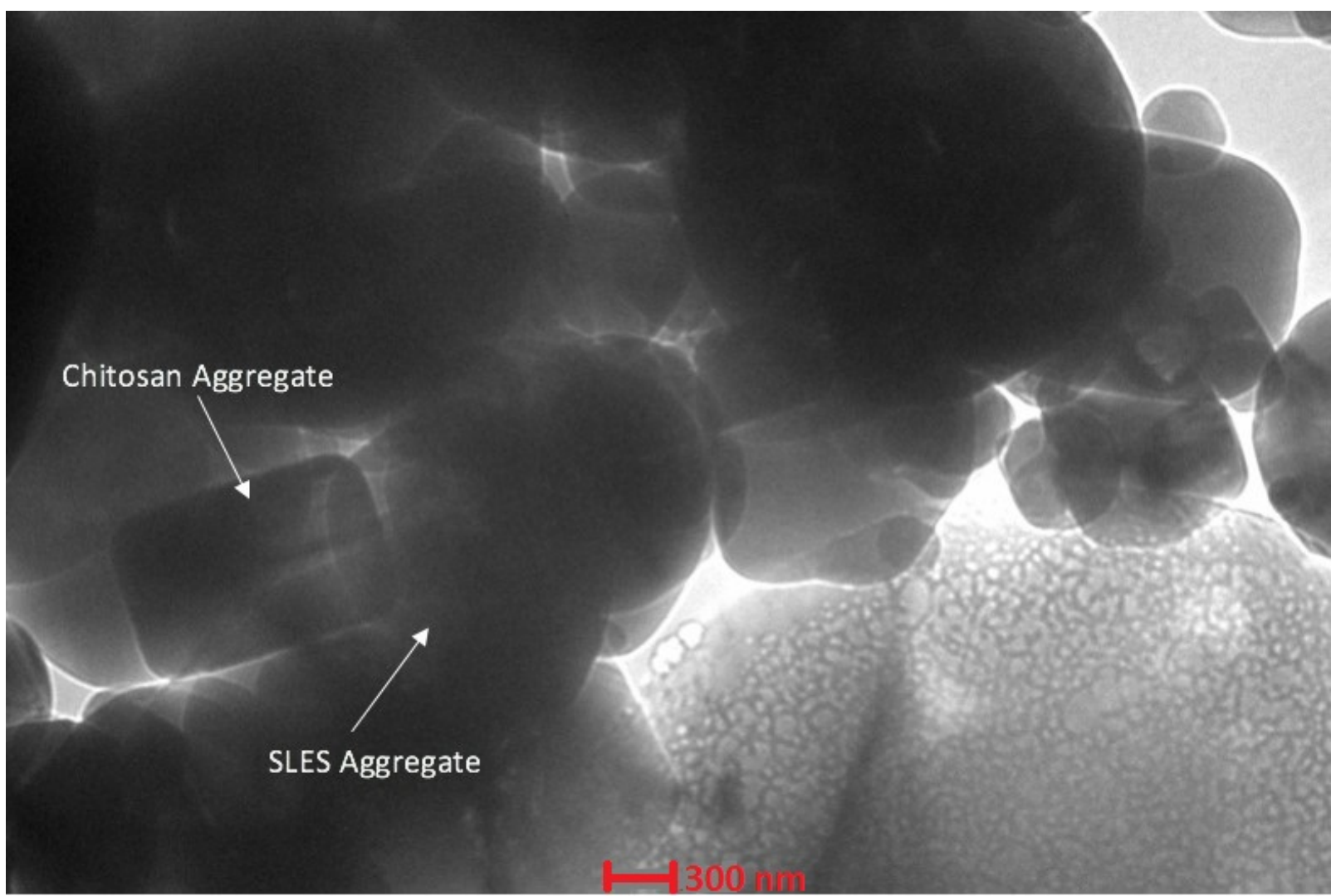

(c)

Figure 4: Cryo-TEM images of (a) 300 ppm chitosan, (b) 400 ppm SLES, and (c) chitosan-SLES complex

and how a clear complex of the two additives was formed. We found that the complex was formed in the macroscale and not in the molecular scale. This observation indicates that the combination of the polymer and surfactant occurred through the combination of the aggregates and not at the molecular level which explains the nonlinear relationship between the chitosan concentration and the complex rheological properties. We believe that the high polarity of the two additives created rigid aggregates that allow macrointeraction only between them and create pseudo- 


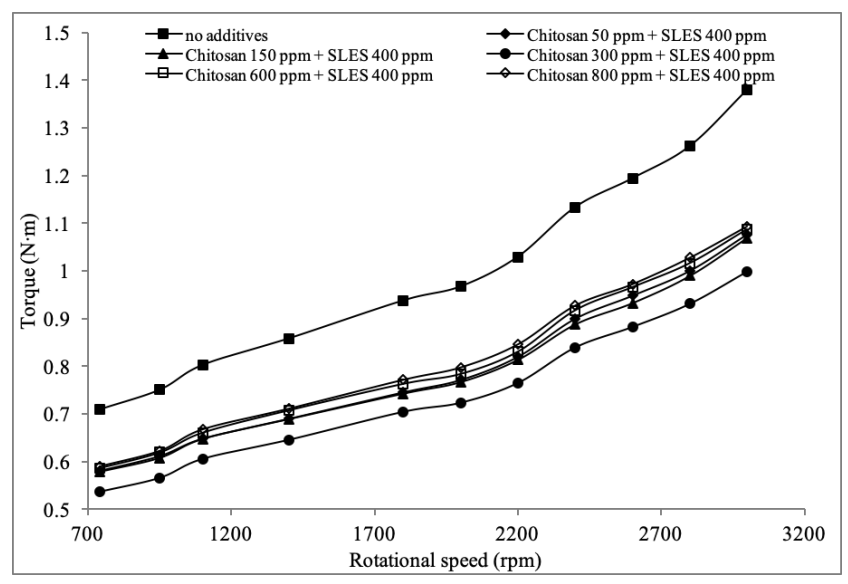

Figure 5: Torque values for chitosan-SLES complexes at different rotational speeds

sites-occupation that prevent further attachment of aggregates.

Figures 5-8 show the drag reduction performance and torque values of the chitosan-SLES complexes. The results are presented on the basis of rotational speed (rpm) instead of Reynolds number (Re) due to the noticeable rheological influence of the additive on the apparent physical properties, such as viscosity. The figures indicate a high drag reduction performance by all the investigated solutions while the torque values when applying the same shear rate (rotational speed) were lower, as shown in Figure 5 and 6 . Increasing the concentration of chitosan in the complex solutions improved the drag reduction performance. However, an adverse effect on the drag reduction efficiency was observed beyond 300 ppm of chitosan. A maximum drag reduction of $47.75 \%$ was observed in the complex with $300 \mathrm{ppm}$ of chitosan mixed at the rotational speed of $3000 \mathrm{rpm}$ and this is expected to be the critical concentration. The maximum drag reduction achieved with the chitosan 300 ppm - SLES 400 ppm complex can also be explained by the rheological and morphological characterization results.

Figures 8 and 9 illustrate the performance of the chitosan-SLES complex in comparison with its pure components (300 ppm chitosan and $400 \mathrm{ppm}$ SLES). The complex solution achieved greater drag reduction performance. The torque value of the complex reduced by $18 \%$ at lower rotational speed compared with those of the pure chitosan and SLES solutions. As demonstrated in Figure 8, at lower rotational speed, the drag reductions achieved by the chitosan-SLES complex increased around $27 \%$ and $39 \%$ compared with the pure chitosan and SLES complexes, respectively. At higher rotational speed (3000 rpm), the drag reductions obtained by the complex were

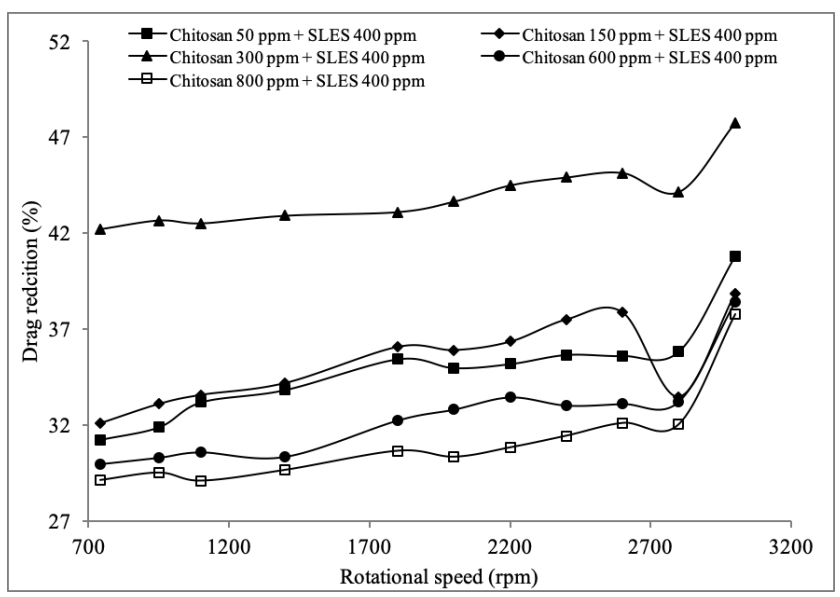

Figure 6: Drag reductions for chitosan-SLES complexes at different rotational speeds

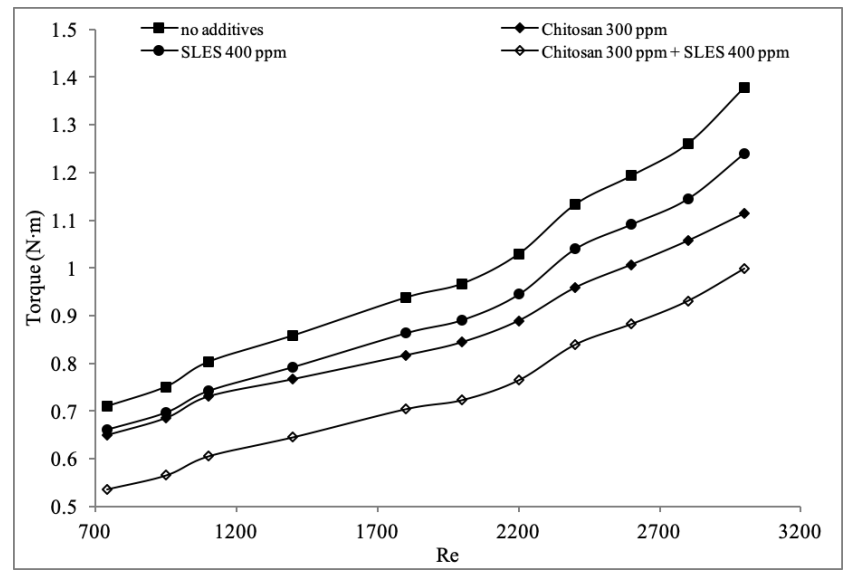

Figure 7: Torque values for chitosan-SLES complexes, pure chitosan, and SLES at different rotational speeds

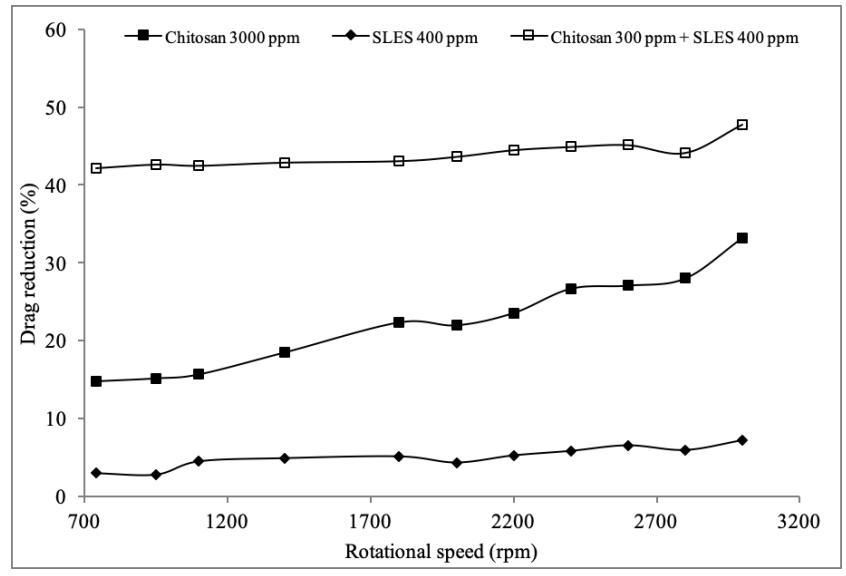

Figure 8: Drag reductions for chitosan-SLES complexes, pure chitosan, and SLES at different rotational speeds 


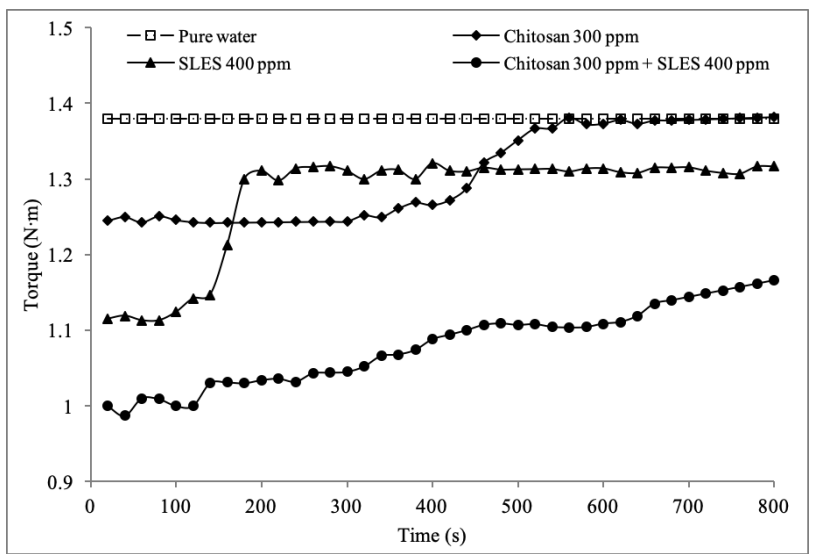

Figure 9: Stability test of chitosan-SLES complex, pure chitosan, and SLES for 800 seconds

about $40 \%$ and $14.54 \%$ compared to those of pure chitosan and SLES, respectively.

The stability of the solutions was investigated by applying the maximum shear force (3000 rpm) for $800 \mathrm{sec}-$ onds. The torque values were monitored throughout the process. Figure 8 shows that the SLES solution had the poorest resistance against shear force while its drag reduction effect was partially lost after 140 seconds and plateaued afterwards. Such behavior is expected since the surfactant molecules forming the aggregates have low resistance to high shear forces, but the drag reduction effect is not completely lost because they can reform into smaller aggregates that can move with the turbulence. The chitosan solution showed a less stable performance because its drag reduction efficiency was completely lost after 560 seconds. This is expected because the mechanical degradation of the polymer molecules is irreversible, i.e., when the chitosan solution is exposed to high shear force its original monomers start to degrade resulting in a permanent loss of its drag reduction effectiveness. The complex solution resistance to shear forces was higher than that of the other two individual additives. The complex linearly lost its resistance to high shear forces and never collapsed when compared with the chitosan solution.

The experimental results indicate a few new interesting points that can lead to a proposal of a controlling mechanism for flow enhancement. The macro-aggregates added new positive advantage in creating a flexible complex with an acceptable resistance toward high shear forces and good drag reduction performance. It is expected that the presence of rigid polar surfactant micelles with their complete and stable spherical shape (as indicated by the cryo-TEM images) will result in combining polymeric molecules in a network-like assemble. Figure 10 shows
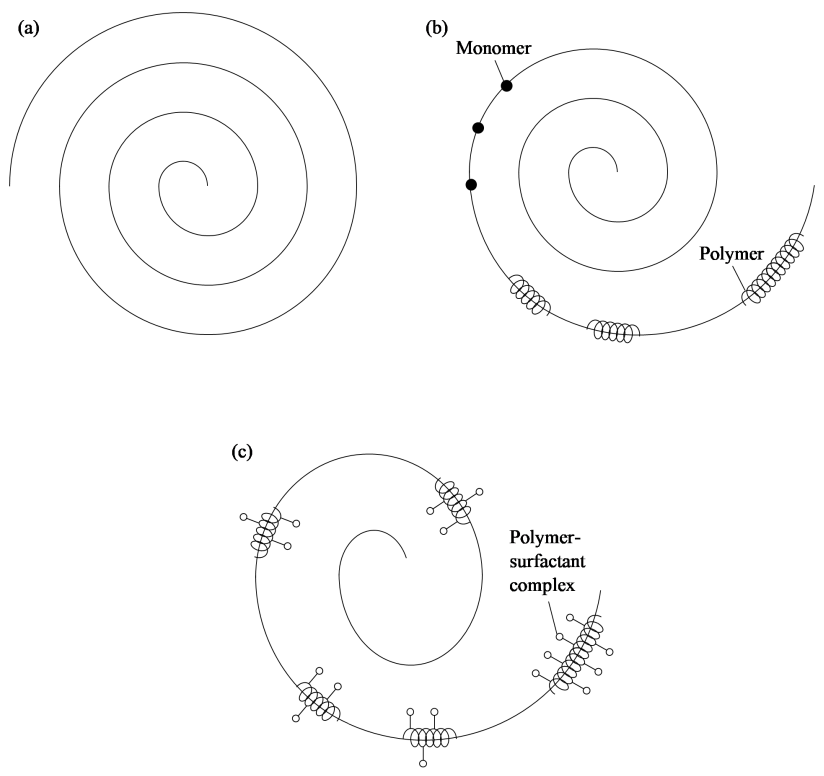

Figure 10: Possible drag reduction mechanism (a) turbulence structure, (b) interaction of polymer molecules and (c) interaction of polymer-surfactant complex molecules with turbulence structure

the expected drag reduction mechanism in the present work. It is believed that the interaction between the turbulence structures (case A, Figure 10a) and the polymeric molecules will result in new rheological properties of the structure itself (new viscoelastic properties) as in case B. In case B, the polymer molecules will be stretched with the globe of liquid forming a turbulence structure. If we assume that this globe is absorbing exactly the same amount of energy absorbed in case A, then the polymeric molecules with viscoelastic properties will prevent it from completing the same shape (absorbing the same amount of energy). On the other hand, the shear force exerted by the turbulence structure itself will stretch the polymeric molecules and break it down to monomers stopping the drag reduction effect of these additives. The introduction of the polymer-surfactant complex will introduce completely new properties to the turbulence system. The interaction between the macrocomplexes and the turbulence will result in a higher turbulence-suppression rate (higher drag reduction) as shown in case C (Figure 10c). Also, the turbulence effect on the stability of the additive will be lower due to the macrointeraction between the polymer and the surfactant molecules, and applying a higher shear rate will break the complexes into smaller macrostructures that maintain the drag reduction performance at the high shear rate without being degraded over time like the polymeric additives. 


\section{Conclusions}

The present work introduces a new organic polymersurfactant complex as a drag reducing agent. The new complex was rheologically and morphologically examined, and the modification of apparent physical properties was observed when compared with the individual additives solutions. The new complex also showed excellent viscoelastic properties which reflect the potential for good drag reduction performance. This was experimentally proven when the solutions were tested with the rotating disk apparatus under severe shear rates. A maximum drag reduction of $47.75 \%$ was obtained by the complex containing $300 \mathrm{ppm}$ of chitosan at the rotational speed of $3000 \mathrm{rpm}$. Unique macrocomplexes that occupied part of the polymer active sites were observed via the cryoTEM images of the polymer-surfactant complex. Finally, we believe that the interaction between the soluble additives and its macrocomplexes with the coherent turbulence structures will result in suppressing the turbulence structures (reduce the power losses).

Acknowledgement: We wish to show our deepest appreciations to the Ministry of Higher Education Malaysia for the financial support of this project through the Fundamental Research Grant Scheme (FRGS/1/2016/TK02/UMP/02/1).

\section{References}

[1] Sun Y., Wu Q., Wei M., Bai B., Ma Y., Experimental study of friction reducer flows in microfracture, Fuel. 2014,131, 28-35.

[2] Le Brun N., Zadrazil I., Norman L., Bismarck A., Markides C.N., On the drag reduction effect and shear stability of improved acrylamide copolymers for enhanced hydraulic fracturing, Chem. Eng. Sci., 2016, 146, 135-143.

[3] Eshrati M., Al-Wahaibi T., Al-Hashmi AR., Al-Wahaibi Y., AlAjmi A., Abubakar A., Experimental study of drag reduction of polymer-polymer mixtures in horizontal dispersed oil-water flow, Exp. Therm. Fluid Sci., 2017, 83(Supplement C), 169-176.

[4] Gu W., Wang D., Turbulence transport of surfactant solution flow during drag reduction degeneration, J, Hydrodyn, Ser B., 2012, 24(4), 479-487.

[5] Cai S., Higuchi Y., Drag-reduction behavior of an unusual nonionic surfactant in a circular pipe turbulent flow, J. Hydrodyn. Ser B., 2014, 26(3), 400-405.

[6] Cai S., Drag reduction of a cationic surfactant solution and its shear stress relaxation, J. Hydrodyn. Ser B., 2012, 24(2), 202206.

[7] Al-Sarkhi A., Drag reduction with polymers in gas-liquid/liquidliquid flows in pipes: A literature review, J. Nat. Gas Sci. Eng., 2010, 2(1), 41-48.
[8] Burger E.D., Munk W.R., Wahl H.A., Flow increase in the trans alaska pipeline through use of a polymeric drag-reducing additive, J. Pet. Technol., 1982, 34(2), 377-386.

[9] Li F.C., Yu B., Wei J.J., Kawaguchi Y., Turbulent drag reduction by surfactant additives, 2011, Wiley, Singapore.

[10] Al-Sarkhi A., Hanratty T.J., Effect of pipe diameter on the performance of drag-reducing polymers in annular gas-liquid flows, Chem. Eng. Res. Des., 2001, 79(4), 402-408.

[11] Mavros P., Ricard A., Xuereb C., Bertrand J., A study of the effect of drag-reducing surfactants on flow patterns in stirred vessels, Chem. Eng. Res. Des., 2011, 89(1), 94-106.

[12] Karami H.R., Mowla D., Investigation of the effects of various parameters on pressure drop reduction in crude oil pipelines by drag reducing agents, J. Nonnewton Fluid Mech., 2012, 177-178, 37-45.

[13] Vlachogiannis M., Hanratty T.J., Influence of wavy structured surfaces and large scale polymer structures on drag reduction, Exp. Fluids., 2004, 36(5), 685-700.

[14] Zhang K., Lim G.H., Choi H.J., Mechanical degradation of watersoluble acrylamide copolymer under a turbulent flow: Effect of molecular weight and temperature, J. Ind. Eng. Chem., 2016, 33, 156-161.

[15] Nsom B., Latrache N., Measurement of drag reduction in dilute polymer solution using triboelectric effect, Appl. Rheol., 2018, 28, 25922.

[16] Abubakar A., Al-Wahaibi T., Al-Wahaibi Y., Al-Hashmi .AR., AlAjmi A., Roles of drag reducing polymers in single- and multiphase flows, Chem. Eng. Res. Des., 2014, 92(11), 2153-2181.

[17] Al-Sarkhi A., Effect of mixing on frictional loss reduction by drag reducing polymer in annular horizontal two-phase flows, Int. J. Multiph. Flow, 2012, 39, 186-192.

[18] Resende P.R., Kim K., Younis B.A., Sureshkumar R., Pinho F.T., A FENE-P $k-\epsilon$ turbulence model for low and intermediate regimes of polymer-induced drag reduction, J. Nonnewton Fluid Mech., 2011, 166(12), 639-660.

[19] Marhefka J., Kameneva M., Natural drag-reducing polymers: Discovery, characterization and potential clinical applications, Fluids, 2016, 1(2), 6.

[20] Soares E.J., Sandoval G.A.B., Silveira L., Pereira A.S., Trevelin R., Thomaz F., Loss of efficiency of polymeric drag reducers induced by high Reynolds number flows in tubes with imposed pressure, Phys, Fluids, 2015, 27(12), 125105.

[21] Kaur H., Singh G., bt. Jaafar A., Study of drag reduction ability of naturally produced polymers from a local plant source, International Petroleum Technology Conference. 2013, Beijing, China.

[22] Hong C.H., Choi H.J., Zhang K., Renou F., Grisel M., Effect of salt on turbulent drag reduction of xanthan gum, Carbohydr. Polym., 2015, 121, 342-347.

[23] Tian M. et al., Rheological and drag reduction properties of hydroxypropyl xanthan gum solutions, Chinese J. Chem. Eng., 2015, 23(9), 1440-1446.

[24] Eshghinejadfard A., Sharma K., Thévenin D., Effect of polymer and fiber additives on pressure drop in a rectangular channel, J. Hydrodyn. Ser. B., 2017, 29(5), 871-878.

[25] Shit S.C., Shah P.M., Edible polymers: Challenges and opportunities, J. Polym., 2014, 2014, 13.

[26] Patil A., Ferritto M.S., Polymers for personal care and cosmetics, Vol. 1148, 2013, ACS Symposium Series. American Chemical Society, Washingtion DC. 
[27] Abdulbari H.A., Ahmad M.A., Yunus R.B.M., Formulation of okranatural mucilage as drag reducing agent in different size of galvanized iron pipes in turbulent water flowing system, J. Appl. Sci., 2010, 10(23), 3105-3110.

[28] Abdulbari H.A., Kamarulizam N.S., Nour A.H., Grafted natural polymer as new drag reducing agent: An experimental approach, Chem. Ind. Chem. Eng. Q., 2012, 18(3), 361-371.

[29] Abdulbari H.A., Letchmanan K., Yunus R.M., Drag reduction characteristics using aloe vera natural mucilage: An experimental study, J. Appl. Sci., 2011, 11, 1039-1043.

[30] Abdulbari H.A., Ling F.W.M., Hibiscus mucilage for enhancing the flow in blood-stream-like microchannel system, Chem. Eng. Commun., 2017, 204(11), 1282-12.98.

[31] Ling F.W.M., Abdulbari H.A., Drag reduction by natural polymeric additives in PMDS microchannel: Effect of types of additives, MATEC Web Conf., 2017, 111, 1001.

[32] Matras Z., Kopiczak B., Intensification of drag reduction effect by simultaneous addition of surfactant and high molecular polymer into the solvent, Chem. Eng. Res. Des., 2015, 96, 35-42.

[33] Antonietti M., Conrad J., Thun̈emann A., Polyelectrolytesurfactant complexes: a new type of solid, mesomorphous material, Macromolecules, 1994, 27, 6007-6011.

[34] Sokolov E.L., Yeh F., Khokhlov A., Grinberg V.Y., Chu B., Nanostructure formation in polyelectrolyte-surfactant complexes, J. Phys. Chem. B, 1998, 102(37), 7091-7098.

[35] Ilekti P., Martin T., Cabane B., Piculell L., Effects of polyelectrolytes on the structures and interactions of surfactant aggregates, J. Phys.. Chem B, 1999, 103(45), 9831-9840.
[36] Bari H.A.A., Mohamad N.K., Mohd N., Nour A.H., Effect of chitosan solution in turbulent drag reduction in aqueous media flow, Sci. Res. Essays, 2011, 6(14), 3058-3064.

[37] Abdulbari H.A., Salleh M.A.M., Rashed M.K., Ismail M.H.S., The effect of passive-active interaction method on drag reduction performance in rotating disk apparatus, Indian J. Sci. Technol., 2017, 10(2)

[38] Akindoyo E.O., Abdulbari H.A., Yousif Z., A dual mechanism of the drag reduction by rigid polymers and cationic surfactant: Complex and nanofluids of xanthan gum and hexadecyl trimethyl ammonium chloride, Int. J. Res. Eng. Technol., 2015, 4(2), 84-93.

[39] Xu X., Chen J., An L., Shear thinning behavior of linear polymer melts under shear flow via nonequilibrium molecular dynamics, J. Chem. Phys., 2014, 140(17), 174902.

[40] Kröger M., Shortest multiple disconnected path for the analysis of entanglements in two- and three-dimensional polymeric systems, Comput. Phys. Commun., 2005, 168(3), 209-232.

[41] Everaers R., Sukumaran S.K., Grest G.S., Svaneborg C., Sivasubramanian A., Kremer K., Rheology and microscopic topology of entangled polymeric liquids, Science. 2004, 303(5659), 823826.

[42] Moraes I.C.F., Fasolin L.H., Cunha R.L., Menegalli F.C., Dynamic and steady-shear rheological properties of xanthan and guar gums dispersed in yellow passion fruit pulp (Passiflora edulis f. flavicarpa), Brazilian J. Chem. Eng., 2011, 28(3), 483-494. 